\title{
Solvable Nonlinear Evolution PDEs in Multidimensional Space*
}

\author{
Francesco CALOGERO ${ }^{\dagger}$ and Matteo SOMMACAL $\ddagger \S$ \\ † Dipartimento di Fisica, Università di Roma "La Sapienza", \\ Istituto Nazionale di Fisica Nucleare, Sezione di Roma, \\ P.le Aldo Moro 2, 00185 Rome, Italy \\ E-mail: francesco.calogero@roma1.infn.it,francesco.calogero@uniroma1.it \\ $\ddagger$ Laboratoire J.-L. Lions, Université Pierre et Marie Curie, Paris VI, \\ 175 Rue du Chevaleret, 75013 Paris, France (until October 30th, 2006) \\ $\S$ Dipartimento di Matematica, Università di Perugia, \\ Via Vanvitelli 1, 06123 Perugia, Italy (from November 1st, 2006) \\ E-mail: matteo.sommacal@pg.infn.it
}

Received October 31, 2006; Published online December 08, 2006

Original article is available at http://www.emis.de/journals/SIGMA/2006/Paper088/

\begin{abstract}
A class of solvable (systems of) nonlinear evolution PDEs in multidimensional space is discussed. We focus on a rotation-invariant system of PDEs of Schrödinger type and on a relativistically-invariant system of PDEs of Klein-Gordon type. Isochronous variants of these evolution PDEs are also considered.
\end{abstract}

Key words: nonlinear evolution PDEs in multidimensions; solvable PDEs; NLS-like equations; nonlinear Klein-Gordon-like equations; isochronicity

2000 Mathematics Subject Classification: 35G25; 35Q40; 37M05

This article is dedicated to the memory of Vadim Kuznetsov, with whom we spent several happy days during a Gathering of Scientists held at the Centro Internacional de Ciencias in Cuernavaca, and as well when he visited us in Rome, and when we met at several other meetings.

\section{Introduction}

Over a decade ago a class of C-integrable - i.e., solvable via a Change of variables - systems of PDEs in multidimensional space were identified [2]. (A problem involving nonlinear PDEs is considered solvable if its solution can be obtained by performing algebraic operations - such as finding the zeros of a given polynomial - and by solving linear PDEs; of course only seldom these operations can be performed explicitly.) In the present paper - motivated by the scarcity of solvable models of nonlinear evolution PDEs in multidimensions hence by the interest of any such model - we study (a subclass of) these solvable PDEs in more detail than it was done hitherto. We focus mainly on the system of PDEs of Schrödinger type

$$
i \psi_{n, t}-\Delta \psi_{n}+W(\vec{r}) \psi_{n}=2 \sum_{m=1, m \neq n}^{N} \frac{a+b \psi_{n} \psi_{m}-\left(\vec{\nabla} \psi_{n}\right) \cdot\left(\vec{\nabla} \psi_{m}\right)}{\psi_{n}-\psi_{m}}
$$

^This paper is a contribution to the Vadim Kuznetsov Memorial Issue "Integrable Systems and Related Topics". The full collection is available at http://www.emis.de/journals/SIGMA/kuznetsov.html 
which is rotation-invariant if $W(\vec{r})=W(r)$, and on the relativistically-invariant system of PDEs of Klein-Gordon type

$$
\psi_{n, t t}-\Delta \psi_{n}+M^{2} \psi_{n}=2 \sum_{m=1, m \neq n}^{N} \frac{a+b \psi_{n} \psi_{m}+\psi_{n, t} \psi_{m, t}-\left(\vec{\nabla} \psi_{n}\right) \cdot\left(\vec{\nabla} \psi_{m}\right)}{\psi_{n}-\psi_{m}}
$$

Notation. Here and throughout $N$ is an arbitrary positive integer $(N \geq 2)$; the index $n$, as well as other analogous indices (see below), range generally from 1 to $N$; the dependent variables $\psi_{n} \equiv \psi_{n}(\vec{r}, t)$ are generally considered complex (although this is only mandatory for the first, (1.1), of these two systems of PDEs); the space variable $\vec{r}$ is a (real) $S$-vector (with $S$ arbitrary: for $S=3, \vec{r} \equiv(x, y, z))$, and $r$ indicates its modulus, $r^{2}=\vec{r} \cdot \vec{r}$; a dot sandwiched among two $S$-vectors denotes the standard (rotation-invariant) scalar product (for instance, for $\left.S=3, \vec{r} \cdot \vec{r}=x^{2}+y^{2}+z^{2}\right)$; the "potential" $W(\vec{r})$ is an arbitrary function of the spatial coordinate $\vec{r}$ (we will often assume that it only depends on the modulus $r$ of the $S$-vector $\vec{r}$ ); the constants $a$ and $b$, as well as the "mass" parameter $M$, are arbitrary (they might also vanish); $t$ is the (real) time variable; subscripted independent variables always denote partial differentiations; $\vec{\nabla}$ respectively $\Delta \equiv \vec{\nabla} \cdot \vec{\nabla}$ are the gradient respectively the Laplace operator in $S$-dimensional space. Isochronous variants of these evolution PDEs are also considered.

In the following Section 2 we review tersely the general class of PDEs treatable in this manner and the technique to solve them. In Sections 3 respectively 4 we treat in some detail the PDEs (1.1) respectively (1.2), describing various properties of their solutions and reporting some representative examples, and we exhibit their isochronous versions. In Section 5 we take advantage of the electronic format of this article to present a few animations displaying visually the time evolution of a few solutions of some of these solvable (systems of) nonlinear evolution PDEs: in this article we restrict these presentations to very few cases, all with space dimensionality less than three (we are of course aware that the three-dimensional case is probably the most interesting one - since we seem to live in a three-dimensional world - but the presentation of animations in a three-dimensional context is somewhat more tricky and we therefore postpone the display of such examples to future articles we hope to issue soon). In Section 6 we outline future directions of research.

\section{A class of solvable (systems of) PDEs in multidimensional space}

In this section we review tersely the basic idea allowing to identify a class of solvable nonlinear evolution PDEs. The interested reader will find a more detailed treatment in the paper where this approach was introduced [1], and especially in [3] where this method is treated in considerable detail: see Section 2.3 of this book, and other references quoted there in Section 2.N. These treatments focussed however on ODEs rather than PDEs: the extension to PDEs is rather straightforward, although it took some time to realize its feasibility [2] (see also Exercise 2.3.4.2-5 in $[3])$.

Let $\Psi(\psi ; \vec{r}, t)$ be a (time- and space-dependent) monic polynomial of degree $N$ in the variable $\psi$, and denote by $\psi_{n}(\vec{r}, t)$ its $N$ zeros and by $\varphi_{m}(\vec{r}, t)$ its $N$ coefficients:

$$
\begin{aligned}
& \Psi(\psi ; \vec{r}, t)=\prod_{n=1}^{N}\left[\psi-\psi_{n}(\vec{r}, t)\right] \\
& \Psi(\psi ; \vec{r}, t)=\psi^{N}+\sum_{m=1}^{N} \varphi_{m}(\vec{r}, t) \psi^{N-m} .
\end{aligned}
$$


Assume then that the time evolution of the dependent variable $\Psi$ - hence its dependence on the time and space independent variables $t$ and $\vec{r}$, as well as its dependence on the independent variable $\psi$ - is characterized by a linear evolution PDE, which must of course be consistent with the fact that $\Psi$ is, for all time, a monic polynomial of degree $N$ in $\psi$, see (2.1). The linear character of this PDE entails that the time-evolution of the $N$ coefficients $\varphi_{m}(\vec{r}, t)$, see $(2.1 \mathrm{~b})$, is as well linear, possibly explicitly solvable (see below). On the other hand the corresponding time evolution of the $N$ zeros $\psi_{n}(\vec{r}, t)$ will be nonlinear, due to the nonlinear character of the mapping relating the $N$ zeros $\psi_{n}(\vec{r}, t)$ to the $N$ coefficients $\varphi_{m}(\vec{r}, t)$ of the polynomial $\Psi(\psi ; \vec{r}, t)$, see (2.1). These (systems of) nonlinear evolution PDEs satisfied by the dependent variables $\psi_{n}(\vec{r}, t)$ are those referred to in the title of this paper: they are indeed generally solvable by taking advantage of the mapping, see (2.1), relating the $N$ dependent variables $\psi_{n}(\vec{r}, t)$ to the $N$ functions $\varphi_{m}(\vec{r}, t)$.

In particular it is easily seen (using, if need be, the formulas provided in Section 2.3 of [3]), that to the evolution PDE

$$
i \Psi_{t}-\Delta \Psi-V(\vec{r})\left(\psi \Psi_{\psi}-N \Psi\right)+a \Psi_{\psi \psi}+b\left[\psi^{2} \Psi_{\psi \psi}-N(N-1) \Psi\right]=0
$$

satisfied by $\Psi(\psi ; \vec{r}, t)$, there corresponds for the $N$ zeros $\psi_{n}(\vec{r}, t)$, see $(2.1 \mathrm{a})$, just the system of nonlinear evolution PDEs of Schrödinger type (1.1) with

$$
W(\vec{r})=V(\vec{r})-2(N-1) b,
$$

while the corresponding evolution of the $N$ coefficients $\varphi_{m}(\vec{r}, t)$, see $(2.1 \mathrm{~b})$, is clearly given by the system of linear evolution PDEs

$$
\begin{aligned}
i \varphi_{m, t} & -\Delta \varphi_{m}+[W(\vec{r})-b(m+3)] m \varphi_{m} \\
& =-a(N-m+2)(N-m+1) \varphi_{m-2}, \quad m=1, \ldots, N
\end{aligned}
$$

with $\varphi_{-1}=0$ and $\varphi_{0}=1$ (see $(2.1 \mathrm{~b})$ ). Note that this system of linear PDEs is decoupled if the constant $a$ vanishes; as we shall see in the following section, it can also be replaced by a decoupled system if the constant $a$ does not vanish, $a \neq 0$, but the potential $W(\vec{r})$ is constant, $W(\vec{r})=C$, see Section 3.

Likewise to the evolution PDE

$$
\Psi_{t t}-\Delta \Psi-\mu^{2}\left[\psi \Psi_{\psi}-N \Psi\right]+a \Psi_{\psi \psi}+b\left[\psi^{2} \Psi_{\psi \psi}-N(N-1) \Psi\right]=0,
$$

there corresponds for the $N$ zeros $\psi_{n}(\vec{r}, t)$ just the system of nonlinear evolution PDEs of Klein-Gordon type (1.2) with

$$
M^{2}=\mu^{2}-2(N-1) b,
$$

while the corresponding evolution of the $N$ coefficients $\varphi_{m}(\vec{r}, t)$ is clearly given by the system of linear evolution PDEs

$$
\begin{aligned}
& \varphi_{m, t t}-\Delta \varphi_{m}+\left[M^{2}-b(m+3)\right] m \varphi_{m} \\
& \quad=-a(N-m+2)(N-m+1) \varphi_{m-2}, \quad m=1, \ldots, N,
\end{aligned}
$$

again with $\varphi_{-1}=0$ and $\varphi_{0}=1$ (see $(2.1 \mathrm{~b})$ ): this system is decoupled if the constant $a$ vanishes, and can be replaced by a decoupled system even if $a$ does not vanish, see Section 4.

Having conveyed, tersely but hopefully clearly, the main idea of this approach to identify solvable systems of nonlinear evolution PDEs, we turn, in the next two sections, to the study of the two systems of nonlinear evolution PDEs (1.1) and (1.2). 


\section{Solvable system of nonlinear PDEs of Schrödinger type}

In this section we investigate the system of nonlinear evolution equations (1.1), firstly by analytic techniques and subsequently by reporting some of its solutions in a representative set of cases.

Remark 3.1. The "mean field" $\bar{\psi}(\vec{r}, t)$

$$
\bar{\psi}(\vec{r}, t)=\frac{1}{N} \sum_{n=1}^{N} \psi_{n}(\vec{r}, t)
$$

satisfies the linear Schrödinger equation

$$
i \bar{\psi}_{t}-\Delta \bar{\psi}+W(\vec{r}) \bar{\psi}=0 .
$$

Proof. Sum the nonlinear evolution PDEs (1.1) over $n$ from 1 to $N$, use (3.1) in the left-hand side, and notice that the double sum in the right-hand side vanishes due to the antisymmetry of the summand under the exchange of the two dummy indices $n$ and $m$.

Remark 3.2. If all the $N$ coefficients $\varphi_{m}$ vanish, $\varphi_{m}=0$, then $\Psi=\psi^{N}$ (see (2.1b)), hence all its $N$ zeros $\psi_{n}$ correspondingly vanish, $\psi_{m}=0$ (and more generally: if the first $M$ coefficients $\varphi_{m}$ vanish, $\varphi_{m}=0$ for $m=1, \ldots, M$, then $M$ of the $N$ zeros of $\Psi$ vanish). Hence to a set of localized solutions $\psi_{n}(\vec{r}, t)$ of the system of nonlinear evolution equations (1.1), characterized by the asymptotic conditions

$$
\lim _{r \rightarrow \infty}\left[\psi_{n}(\vec{r}, t)\right]=0, \quad n=1, \ldots, N,
$$

there correspond a set of localized solutions of the system of linear Schrödinger PDEs (2.4) (with (2.3)), characterized by the analogous asymptotic conditions

$$
\lim _{r \rightarrow \infty}\left[\varphi_{n}(\vec{r}, t)\right]=0, \quad n=1, \ldots, N
$$

and, of course, viceversa, namely clearly (3.2b) entails (3.2a). However when two different components, $\psi_{n}(\vec{r}, t)$ and $\psi_{m}(\vec{r}, t)$ with $n \neq m$, of the system of nonlinear evolution equations (1.1) are equal, $\psi_{n}(\vec{r}, t)=\psi_{m}(\vec{r}, t)$, clearly this system runs into a singularity due to the vanishing of one of the denominators in the right-hand side of (1.1); and this situation gets even worst if the two different components, $\psi_{n}(\vec{r}, t)$ and $\psi_{m}(\vec{r}, t)$ with $n \neq m$, both vanish, $\psi_{n}(\vec{r}, t)=\psi_{m}(\vec{r}, t)=0$. Hence in all the examples considered below we shall try and avoid this problem, and in particular we shall focus, rather than on localized solutions $\psi_{n}(\vec{r}, t)$ that vanish asymptotically (see (3.2a)), either on solutions that oscillate asymptotically, or on solutions that are asymptotically constant,

$$
\lim _{r \rightarrow \infty}\left[\psi_{n}(\vec{r}, t)\right]=a_{n} \quad \text { with } a_{n} \neq a_{m} \quad \text { if } \quad n \neq m
$$

Note that these asymptotic values $a_{n}$ might depend on the direction along which the space coordinate $\vec{r}$ diverges.

Remark 3.3. If the potential $V(r)$ is constant,

$$
V(r)=B,
$$

(entailing that $W(r)$ is as well constant,

$$
W(r)=B-2(N-1) b,
$$


see (2.3)), it may be convenient to replace the expression (2.1b) of the monic polynomial $\Psi(\psi ; \vec{r}, t)$ in terms of its coefficients $\varphi_{m}(\vec{r}, t)$ by the following representation:

$$
\begin{aligned}
& \Psi(\psi ; \vec{r}, t)=\frac{C_{N}^{\gamma}(c \psi)}{k_{N} c^{N}}+\sum_{m=1}^{N} \chi_{m}(\vec{r}, t) \frac{C_{N-m}^{\gamma}(c \psi)}{k_{N-m} c^{N-m}}, \\
& c=\left(-\frac{b}{a}\right)^{1 / 2}, \\
& \gamma=-\frac{B+b}{2 b},
\end{aligned}
$$

where the polynomial $C_{\ell}^{\gamma}(z)$, of degree $\ell$, is the standard Gegenbauer polynomial [5], satisfying the ODE

$$
\left(1-z^{2}\right) C_{l}^{\gamma \prime \prime}(z)-(2 \gamma+1) z C_{l}^{\gamma \prime}(z)+\ell(\ell+2 \gamma) C_{l}^{\gamma}(z)=0
$$

(where appended primes denote derivatives with respect to the argument of the function they are appended to, in this case with respect to $z$ ), and being characterized by the asymptotic behavior

$$
\lim _{z \rightarrow \infty}\left[\frac{C_{l}^{\gamma}(z)}{k_{\ell}}\right]=1, \quad \text { where } \quad k_{\ell}=\frac{2^{\ell} \Gamma(\gamma+\ell)}{\ell ! \Gamma(\gamma)} .
$$

Then the requirement that $\Psi(\psi ; \vec{r}, t)$ satisfy the PDE (2.2) with (3.4), and therefore that its $N$ zeros $\psi_{n}(\vec{r}, t)$ satisfy the system of nonlinear PDEs (1.1) with (3.4), entails that the $N$ coefficients $\chi_{m}(\vec{r}, t)$ satisfy the (system of decoupled) linear Schrödinger PDEs (with constant potentials)

$$
i \chi_{m, t}-\Delta \chi_{m}+[B-b(2 N-m-1)] m \chi_{m}=0, \quad m=1, \ldots, N .
$$

The investigation of the limiting cases in which some coefficients vanish, and therefore a different set of classical polynomials come into play in place of the Gegenbauer polynomials, is left as an exercise for the diligent reader.

The nonlinear mapping among the $N$ dependent variables $\psi_{n}(\vec{r}, t)$ satisfying the system of nonlinear PDEs (1.1) and the $N$ functions $\varphi_{m}(\vec{r}, t)$ respectively $\chi_{m}(\vec{r}, t)$ satisfying the system of linear PDEs (2.4) respectively (3.6), entailed by the simultaneous validity of (2.1a) and (2.1b) respectively (3.5), is the key to the solvability of the system of nonlinear PDEs (1.1). This can be taken advantage of in two ways: to solve the initial-value problem for the system of nonlinear PDEs (1.1), or to manufacture special, possibly quite explicit, solutions of this system of nonlinear PDEs.

\subsection{Solution of the initial-value problem for the system of nonlinear Schrödinger-like PDEs (1.1)}

The initial-value problem consists in the determination of the solution $\psi_{n}(\vec{r}, t), n=1, \ldots, N$, of the system of Schrödinger-like nonlinear PDEs (1.1) corresponding to assigned initial data $\psi_{m}(\vec{r}, 0), m=1, \ldots, N$.

The first step is to determine the corresponding initial data $\varphi_{m}(\vec{r}, 0)$ of the system of Schrödinger-like linear PDEs (2.4). This is achieved by solving for the $N$ functions $\varphi_{m}(\vec{r}, 0)$ the system

$$
\psi^{N}+\sum_{m=1}^{N} \varphi_{m}(\vec{r}, 0) \psi^{N-m}=\prod_{n=1}^{N}\left[\psi-\psi_{n}(\vec{r}, 0)\right],
$$


entailed by the simultaneous validity of $(2.1 \mathrm{a})$ and $(2.1 \mathrm{~b})$ at $t=0$. This amounts to the determination of the $N$ coefficients $\varphi_{m}$ of a monic polynomial given its $N$ zeros $\psi_{n}$; the relevant, explicit formulas are of course well-known:

$$
\varphi_{1}(\vec{r}, 0)=-\sum_{n=1}^{N} \psi_{n}(\vec{r}, 0), \quad \varphi_{2}(\vec{r}, 0)=\sum_{n, m=1, m \neq n}^{N} \psi_{n}(\vec{r}, 0) \psi_{m}(\vec{r}, 0),
$$

and so on, up to

$$
\varphi_{N}(\vec{r}, 0)=(-)^{N} \prod_{n=1}^{N} \psi_{n}(\vec{r}, 0) .
$$

The second step is to solve the initial-value problem for the system of evolution PDEs (2.4), obtaining thereby its solution $\varphi_{m}(\vec{r}, t)$ at time $t$. The linear character of this (coupled) system of evolution PDEs, (2.4), provides the main simplification; of course an explicit solution is only possible for special choices of the potential $W(\vec{r})$.

The third step is to obtain the solution $\psi_{n}(\vec{r}, t)$ from the, now assumedly known, functions $\varphi_{m}(\vec{r}, t)$, via the relation

$$
\prod_{n}^{N}\left[\psi-\psi_{n}(\vec{r}, t)\right]=\psi^{N}+\sum_{m=1}^{N} \varphi_{m}(\vec{r}, t) \psi^{N-m},
$$

again entailed by the simultaneous validity of (2.1a) and (2.1b), but now at time $t$. This amounts of course just to the purely algebraic task of finding the zeros of a given monic polynomial of degree $N$ : an explicit solution is of course only possible for $N \leq 4$.

In the special case of a constant potential $V(r)$, see (3.4), an alternative procedure of solution can be based on the representation (3.5) rather than (2.1b): this eases the second of the steps outlined above, but makes a bit less simple the first step. The diligent reader will easily figure out the relevant details.

\subsection{How to manufacture explicit solutions of the system of nonlinear Schrödinger-like PDEs (1.1)}

Clearly the appropriate strategy - underlining all the examples exhibited below - is to identify an explicit solution $\varphi_{m}(\vec{r}, t)$ of the system of linear evolution PDEs (2.4), and then to obtain the corresponding solution $\psi_{n}(\vec{r}, t)$ of the system of nonlinear evolution PDEs (1.1) via (3.8), namely by identifying the $N$ zeros $\psi_{n}(\vec{r}, t)$ of the monic polynomial of degree $N$ in $\psi$ having the coefficients $\varphi_{m}(\vec{r}, t)$, see $(2.1 \mathrm{~b})$. This can of course be done explicitly only for $N \leq 4$ : not a significant restriction when it comes to the explicit exhibition of examples, which would indeed be impractical for larger values of $N$ (in Section 5 we indeed limit our exhibition of animations to the 3-body case, $N=3$ ).

An alternative route - applicable when the potential is constant, see (3.4) - takes as starting point an explicit solution $\chi_{m}(\vec{r}, t)$ of the (decoupled) system of linear evolution PDEs (3.6), and then obtains the corresponding solution $\psi_{n}(\vec{r}, t)$ of the system of nonlinear evolution PDEs (1.1) via (3.5), namely by identifying the $N$ zeros $\psi_{n}(\vec{r}, t)$ of the monic polynomial of degree $N$ in $\psi$ given by this expression (3.5).

\subsection{Examples}

Example 3.3.1. The simplest example is characterized by the assignment

$$
a=b=0, \quad W(\vec{r})=V(\vec{r})=0,
$$


namely by the system of nonlinear evolution PDEs in one-dimensional space (see (1.1))

$$
i \psi_{n, t}-\Delta \psi_{n}=-2 \sum_{m=1, m \neq n}^{N} \frac{\vec{\nabla} \psi_{n} \cdot \vec{\nabla} \psi_{m}}{\psi_{n}-\psi_{m}}
$$

The corresponding system of linear PDEs satisfied by the coefficients $\varphi_{m}(\vec{r}, t)$ of the polynomial $\Psi(\psi ; \vec{r}, t)$ in the variable $\psi$ of which the solutions $\psi_{n}(\vec{r}, t)$ are the $N$ zeros,

$$
\Psi(\psi ; \vec{r}, t)=\psi^{N}+\sum_{m=1}^{N} \varphi_{m}(\vec{r}, t) \psi^{N-m}=\prod_{n}^{N}\left[\psi-\psi_{n}(\vec{r}, t)\right],
$$

reads as follows (see (2.4)):

$$
i \varphi_{m, t}-\Delta \varphi_{m}=0, \quad m=1, \ldots, N .
$$

A special class of "traveling wave" solutions of these (decoupled) linear PDEs reads

$$
\varphi_{m}(\vec{r}, t)=\varphi_{m}(\vec{r}-\vec{v} t)=A_{m}+B_{m} \exp [-i \vec{v}(\vec{r}-\vec{v} t)],
$$

with $\vec{v}$ an arbitrary real constant $S$-vector and the $2 N$ scalar constants $A_{m}, B_{m}$ also arbitrary (possibly complex). These solutions (which are clearly the most general ones of "traveling wave" character, namely depending on the single $S$-vector $\vec{r}-\vec{v} t$ rather than separately on the $S$-vector space variable $\vec{r}$ and the scalar time variable $t$ ) are not localized: they are constant along the $S-1$ space directions orthogonal to $\vec{v}$, periodic with period $L=|2 \pi / v|$ along the space direction parallel to $\vec{v}$, and periodic in $t$ with period $T=2 \pi v^{-2}$. The corresponding traveling wave solutions $\psi_{n}(x, t)$ of the system of nonlinear PDEs (3.9) are the $N$ zeros of the following polynomial of degree $N$ in the variable $\psi$ :

$$
P_{N}(\psi)+Q_{N-1}(\psi) \exp [-i \vec{v}(\vec{r}-\vec{v} t)]=\prod_{n}^{N}\left[\psi-\psi_{n}(\vec{r}, t)\right],
$$

where $P_{N}(\psi)$ is an arbitrary monic polynomial of degree $N$ and $Q_{N-1}(\psi)$ is an arbitrary polynomial of degree $N-1$,

$$
P_{N}(\psi)=\psi^{N}+\sum_{m=1}^{N} A_{m} \psi^{N-m}, \quad Q_{N-1}(\psi)=\sum_{m=1}^{N} B_{m} \psi^{N-m} .
$$

This entails of course that these solutions are as well of the same traveling wave type, $\psi_{n}(\vec{r}, t)=$ $\psi_{n}(\vec{r}-\vec{v} t)$, with the same periodicity properties described above for $\varphi_{m}(\vec{r}, t)=\varphi_{m}(\vec{r}-\vec{v} t)-$ or possibly with time periods which are integer multiples of that of the coefficients $\varphi_{m}(\vec{r}, t)$ due to the possibility that through the time evolution the zeros of the polynomial (3.13b) exchange their roles; for each component $\psi_{n}(\vec{r}, t)$ this integer multiple cannot of course exceed $N$, while for the entire solution $\left\{\psi_{n}(\vec{r}, t) ; n=1, \ldots, N\right\}$ the period can be somewhat larger - but generally not too much [6].

A solution of this kind (with $S=2$ and $N=3$, and a specific choice of the remaining free parameters) is displayed as an animation in Section 5 .

Another special set of solutions of (3.11) - written below, for simplicity, for the one-dimensional case $(S=1)$ - reads

$$
\varphi_{m}(x, t)=A_{m}+\frac{B_{m}}{\left(t+t_{m}+i \eta_{m}\right)^{1 / 2}} \exp \left[\frac{-i\left(x-x_{m}+i \eta_{m} v_{m}\right)^{2}}{4\left(t+t_{m}+i \eta_{m}\right)}\right],
$$


where the constants $A_{m}$ and $B_{m}$ are arbitrary (possibly complex; but see below for some conditions on the constants $A_{m}$ ), the constants $x_{m}, t_{m}$ and $v_{m}$ are also arbitrary but real, and the constants $\eta_{m}$ are also arbitrary but positive, $\eta_{m}>0$ : it is indeed easily seen that these conditions are sufficient to guarantee that $\varphi_{m}(x, t)$ is nonsingular for all real values of the independent variables $x$ and $t$ and tend asymptotically to the constants $A_{m}$ :

$$
\lim _{x \rightarrow \pm \infty}\left[\varphi_{m}(x, t)\right]=A_{m} .
$$

The corresponding solutions $\psi_{n}(x, t)$ of the system of nonlinear PDEs (3.9) are the $N$ zeros of the following polynomial of degree $N$ in the variable $\psi$ :

$$
\begin{aligned}
\psi^{N}+ & \sum_{m=1}^{N}\left\{A_{m}+\frac{B_{m}}{\left(t+t_{m}+i \eta_{m}\right)^{1 / 2}} \exp \left[\frac{-i\left(x-x_{m}+i \eta_{m} v_{m}\right)^{2}}{4\left(t+t_{m}+i \eta_{m}\right)}\right]\right\} \psi^{N-m} \\
& =\prod_{n=1}^{N}\left[\psi-\psi_{n}(x, t)\right] .
\end{aligned}
$$

Note that this entails that these solutions satisfy the asymptotic property (3.3), with the constants $a_{n}$ being the $N$ zeros of the polynomial, of degree $N$ in the variable $a$,

$$
a^{N}+\sum_{m=1}^{N} A_{m} a^{N-m}=\prod_{n=1}^{N}\left(a-a_{n}\right) .
$$

Of course the constants $A_{m}$ should be assigned so that these $N$ zeros $a_{n}$ are all different, see (3.3).

A solution of this kind (with $N=3$, and a specific choice of all the remaining free parameters) is displayed as an animation in Section 5.

\subsection{Isochronous version of the class of nonlinear PDEs of Schrödinger type}

In this subsection we report, without much commentary, an " $\omega$-modified" version of (a subclass of) the system of nonlinear PDEs of Schrödinger type (1.1), which is characterized by the property to possess an ample class of solutions completely periodic in time with period

$$
T=\frac{2 \pi}{\omega}
$$

(the reason why this is so will be rather obvious from what follows; for more details on, and other examples of, isochronous PDEs obtained in an analogous manner see [7] and Chapter 6 of $[4])$.

We start from the subcase of the system of nonlinear PDEs of Schrödinger type (1.1) with

$$
b=0, \quad W(\vec{r})=0,
$$

and we set

$$
\tilde{\psi}_{n}(\vec{r}, t)=e^{i \lambda \omega t} \psi_{n}(\vec{\rho}, \tau), \quad \vec{\rho} \equiv \vec{\rho}(t)=e^{\frac{i \omega t}{2}} \vec{r}, \quad \tau \equiv \tau(t)=\frac{e^{i \omega t}-1}{i \omega},
$$

with $\omega$ an arbitrary positive constant and $\lambda$ an arbitrary real rational number if $a$ vanishes ( $a=0$, see (1.1) and below), otherwise $\lambda=-1 / 2$. It is then easily seen that the new dependent variables $\tilde{\psi}_{n}(\vec{r}, t)$ satisfy the following system of nonlinear PDEs of Schrödinger type:

$$
i \tilde{\psi}_{n, t}-\Delta \tilde{\psi}_{n}+\lambda \omega \tilde{\psi}_{n}+\frac{\omega}{2} \vec{r} \cdot \vec{\nabla} \tilde{\psi}_{n}=2 \sum_{m=1, m \neq n}^{N} \frac{a-\left(\vec{\nabla} \tilde{\psi}_{n}\right) \cdot\left(\vec{\nabla} \tilde{\psi}_{m}\right)}{\tilde{\psi}_{n}-\tilde{\psi}_{m}} .
$$


Note that this system is autonomous with respect to the time variable, but it features an explicit dependence on the space variable $\vec{r}$ (see the last term in the left-hand side); and it is clearly rotation invariant.

Example 3.4.1. The simplest example is again characterized by the assignment

$$
S=1, \quad a=0,
$$

namely by the system of nonlinear evolution PDEs in one-dimensional space (see (1.1))

$$
i \tilde{\psi}_{n, t}-\tilde{\psi}_{n, x x}+\lambda \omega \tilde{\psi}_{n}+\frac{\omega}{2} x \tilde{\psi}_{n, x}=-2 \sum_{m=1, m \neq n}^{N} \frac{\tilde{\psi}_{n, x} \tilde{\psi}_{m, x}}{\tilde{\psi}_{n}-\tilde{\psi}_{m}} .
$$

A special class of solutions of this system of nonlinear PDEs obtains, via the formula

$$
\begin{aligned}
& \tilde{\psi}_{n}(x, t)=\exp (i \lambda \omega t) \psi_{n}(\xi, \tau), \\
& \xi \equiv \xi(t)=\exp \left(\frac{i \omega t}{2}\right) x, \quad \tau \equiv \tau(t)=\frac{\exp (i \omega t)-1}{i \omega},
\end{aligned}
$$

from the solutions $\psi_{n}(\xi, \tau)$ of $(3.13 \mathrm{a})$, now reading

$$
P_{N}(\psi)+Q_{N-1}(\psi) \exp [-i v(\xi-v \tau)]=\prod_{n}^{N}\left[\psi-\psi_{n}(\xi, \tau)\right],
$$

where $P_{N}(\psi)$ is an arbitrary monic polynomial of degree $N$ and $Q_{N-1}(\psi)$ is an arbitrary polynomial of degree $N-1$, see (3.13b).

Another class of solutions is provided via (3.17) from the solutions $\psi_{n}$ of (3.15), now reading

$$
\begin{aligned}
\psi^{N}+ & \sum_{m=1}^{N}\left\{A_{m}+\frac{B_{m}}{\left(\tau+\tau_{m}+i \eta_{m}\right)^{1 / 2}} \exp \left[\frac{-i\left(\xi-\xi_{m}+i \eta_{m} v_{m}\right)^{2}}{4\left(\tau+\tau_{m}+i \eta_{m}\right)}\right]\right\} \psi^{N-m} \\
& =\prod_{n=1}^{N}\left[\psi-\psi_{n}(\xi, \tau)\right]
\end{aligned}
$$

\section{Solvable systems of nonlinear PDEs of Klein-Gordon type}

In this section we investigate, firstly by analytic techniques and subsequently via the explicit display of a few of its solutions, the system of nonlinear evolution equations of Klein-Gordon type (1.2). The first remarks are analogous to those given in the first part of the preceding section and are therefore reported below without much commentary (their proofs are analogous to those given in the preceding section; we also use occasionally the same notation, confiding that this will cause no misunderstandings).

Remark 4.1. The "mean field" $\bar{\psi}(\vec{r}, t)$ defined by (3.1) satisfies now the linear Klein-Gordon equation

$$
\bar{\psi}_{t t}-\Delta \bar{\psi}+M^{2} \bar{\psi}=0
$$

Remark 4.2. To a set of localized solutions $\psi_{n}(\vec{r}, t)$ of the system of nonlinear evolution equations of Klein-Gordon type (1.2) characterized by the asymptotic conditions (3.2a) there correspond a set of localized solutions of the system of linear Klein-Gordon PDEs (2.6) characterized by the analogous asymptotic conditions (3.2b); and, of course, viceversa. But such localized solutions cause the same kind of problem discussed in Remark 3.1. Hence in all the examples considered below we shall try and avoid this problem, just as indicated in Remark 3.2. 
Remark 4.3. It may be convenient to replace the expression (2.1b) of the monic polynomial $\Psi(\psi ; \vec{r}, t)$ in terms of its coefficients $\varphi_{m}(\vec{r}, t)$ by the representation (3.5a), again with (3.5b) but now with

$$
\gamma=-\frac{M^{2}+b(2 N-m)}{2 b}
$$

instead of (3.5c). Then the requirement that $\Psi(\psi ; \vec{r}, t)$ satisfy the PDE $(2.5)$, and therefore that its $N$ zeros $\psi_{n}(\vec{r}, t)$ satisfy the system of nonlinear PDEs (1.2), entails that the $N$ coefficients $\chi_{m}(\vec{r}, t)$ satisfy the (system of) decoupled linear Klein-Gordon PDEs

$$
\chi_{m, t t}-\Delta \chi_{m}+\left[M^{2}-b(m+3)\right] m \chi_{m}=0, \quad m=1, \ldots, N .
$$

Analogous developments to those reported in the preceding Section 3 in the context of the system of Schrödinger-like nonlinear evolution PDEs (1.1) can now be elaborated in the present context of the system of Klein-Gordon-like nonlinear evolution PDEs (1.2). Our presentation below is more terse than in the preceding section, to avoid repetitions.

\subsection{Solution of the initial-value problem for the system of nonlinear Klein-Gordon-like PDEs (1.2)}

The initial-value problem consists now in the determination of the solution $\psi_{n}(\vec{r}, t)$ of the system of Klein-Gordon-like nonlinear PDEs (1.2) corresponding to assigned initial data $\psi_{m}(\vec{r}, 0)$ and $\psi_{m, t}(\vec{r}, 0), m=1, \ldots, N$.

The first step is to determine the corresponding initial data $\varphi_{m}(\vec{r}, 0)$ and $\varphi_{m, t}(\vec{r}, 0)$ of the system of Klein-Gordon-like linear PDEs (2.6). This is achieved by firstly solving, as above, for the $N$ functions $\varphi_{m}(\vec{r}, 0)$ the system (3.7), and then by solving for the $N$ functions $\varphi_{m, t}(\vec{r}, 0)$ the system

$$
\sum_{m=1}^{N} \varphi_{m, t}(\vec{r}, 0) \psi^{N-m}=-\sum_{n=1}^{N}\left\{\psi_{n, t}(\vec{r}, 0) \prod_{m=1, m \neq n}^{N}\left[\psi-\psi_{m}(\vec{r}, 0)\right]\right\} .
$$

The second step is to obtain the solution $\varphi_{m}(\vec{r}, t)$ at time $t$ of the system of evolution PDEs (2.6). Again, the linear character of this system of evolution PDEs provides the main simplification.

The third step is, as above, to obtain the solution $\psi_{n}(\vec{r}, t)$ from the, now assumedly known, functions $\varphi_{m}(\vec{r}, t)$ via the relation (3.8), amounting again just to the purely algebraic task of finding the $N$ zeros of a given monic polynomial of degree $N$.

An alternative procedure of solution can be based on the representation (3.5a) (with (3.5b) and (4.1) rather than (3.5c)): this eases the second of the steps outlined above, but makes a bit less simple the first step. The diligent reader will easily figure out the relevant details.

\subsection{How to manufacture explicit solutions of the system of nonlinear Klein-Gordon-like PDEs (1.2)}

As above the strategy - that underlies all the examples discussed below - is to identify an explicit solution $\varphi_{m}(\vec{r}, t)$ of the system of linear evolution PDEs (2.6), and then to obtain the corresponding solution $\psi_{n}(\vec{r}, t)$ of the system of nonlinear evolution PDEs (1.2) via (3.8), namely by identifying the $N$ zeros $\psi_{n}(\vec{r}, t)$ of the monic polynomial of degree $N$ in $\psi$ with coefficients $\varphi_{m}(\vec{r}, t)$, see $(2.1 \mathrm{~b})$.

An alternative route takes as starting point an explicit solution $\chi_{m}(\vec{r}, t)$ of the (decoupled) system of linear evolution PDEs (4.2), and then obtains the corresponding solution $\psi_{n}(\vec{r}, t)$ 
of the system of nonlinear evolution PDEs (1.2) by identifying the quantities $\psi_{n}(\vec{r}, t)$ as the $N$ zeros of the monic polynomial of degree $N$ in $\psi$ given by the expression (3.5a) with (3.5b) and (4.1).

\subsection{Examples}

Example 4.3.1. The simplest example is characterized by the assignment

$$
a=b=0, \quad M=0,
$$

namely by the system of nonlinear evolution PDEs (see (1.2))

$$
\psi_{n, t t}-\Delta \psi_{n}=2 \sum_{m=1, m \neq n}^{N} \frac{\psi_{n, t} \psi_{m, t}-\vec{\nabla} \psi_{n} \cdot \vec{\nabla} \psi_{m}}{\psi_{n}-\psi_{m}} .
$$

The corresponding system of linear PDEs satisfied by the coefficients $\varphi_{m}(\vec{r}, t)$ of the polynomial $\Psi(\psi ; \vec{r}, t)$ in the variable $\psi$ of which the solutions $\psi_{n}(\vec{r}, t)$ are the $N$ zeros, see $(3.10)$, reads as follows (see (2.6)):

$$
\varphi_{m, t t}-\Delta \varphi_{m}=0, \quad m=1, \ldots, N .
$$

The general solution of these linear PDEs reads

$$
\varphi_{m}(\vec{r}, t)=\sum_{k=1}^{K} f_{m k}\left(\vec{r}-\vec{u}_{k} t\right),
$$

with the $K N$ functions $f_{m k}(\vec{r})$ arbitrary and the $K$ constant $S$-vectors $\vec{u}_{k}$ having unit length, $u_{k}=1$, but being otherwise arbitrary. Of course these solutions $\varphi_{m}(\vec{r}, t)$ are localized if the arbitrary functions $f_{m k}(\vec{r})$ are themselves localized, but (motivated by Remark 4.2) we shall rather consider solutions that tend asymptotically to nonvanishing asymptotic values; and $\varphi_{m}(\vec{r}, t)$ has the character of a traveling wave if $K=1$.

The corresponding solutions $\psi_{n}(\vec{r}, t)$ of the system of nonlinear PDEs (4.3) are the $N$ zeros of the following polynomial of degree $N$ in $\psi$ :

$$
\Psi(\psi ; \vec{r}, t)=\psi^{N}+\sum_{m=1}^{N} \psi^{N-m} \sum_{k=1}^{K} f_{m k}\left(\vec{r}-\vec{u}_{k} t\right)=\prod_{n}^{N}\left[\psi-\psi_{n}(\vec{r}, t)\right] .
$$

Two solutions of this kind (with $S=2$ respectively $S=1, N=3$, and a specific choice of the remaining free parameters) are displayed as animations in Section 5.

Example 4.3.2. An analogous example - but reported here for simplicity in the two-dimensional case $(S=2)$ - is characterized by the analogous assignment

$$
S=2, \quad a=b=0, \quad M=0,
$$

namely by the system of nonlinear evolution PDEs in two-dimensional space (see (1.1))

$$
\psi_{n, t t}-\psi_{n, x x}-\psi_{n, y y}=2 \sum_{m=1, m \neq n}^{N} \frac{\psi_{n, t} \psi_{m, t}-\psi_{n, x} \psi_{m, x}-\psi_{n, y} \psi_{m, y}}{\psi_{n}-\psi_{m}} .
$$

The corresponding system of (decoupled) PDEs satisfied by the coefficients $\varphi_{m}(x, y, t)$ reads

$$
\varphi_{m, t t}-\varphi_{m, x x}-\varphi_{m, y y}=0 .
$$


A class of regular solutions of this system of PDEs reads

$$
\varphi_{m}(x, y, t)=J_{0}\left(\sqrt{\left(x-x_{m}\right)^{2}+\left(y-y_{m}\right)^{2}}\right)\left[A_{m} \cos (t)+B_{m} \sin (t)\right]+C_{m},
$$

where $A_{m}, B_{m}$ and $C_{m}$ are $3 N$ arbitrary constants (possibly complex), and $J_{0}(r)$ is the zerothorder Bessel function of the first kind. A solution of this kind (with $N=3$, and a specific choice of the remaining free parameters) is displayed as an animation in Section 5.

\subsection{Isochronous version of the class of nonlinear PDEs of Klein-Gordon type}

In this subsection we report, with even less commentary than in the (analogous) Subsection 3.4, an " $\omega$-modified" version of (a subclass of) the system of nonlinear PDEs of Klein-Gordon type (1.2), which is again characterized by the property to possess an ample class of solutions completely periodic in time with period $T$, see (3.16).

Now we start from the subcase of the system of nonlinear PDEs of Klein-Gordon type (1.2) with

$$
b=0, \quad M=0,
$$

and we set

$$
\tilde{\psi}_{n}(\vec{r}, t)=e^{i \lambda \omega t} \psi_{n}(\vec{\rho}, \tau), \quad \vec{\rho} \equiv \vec{\rho}(t)=e^{i \omega t} \vec{r}, \quad \tau \equiv \tau(t)=\frac{e^{i \omega t}-1}{i \omega},
$$

with $\omega$ an arbitrary positive constant and $\lambda$ an arbitrary real rational number if $a$ vanishes ( $a=0$, see (1.2) and below), otherwise $\lambda=-1$. It is then easily seen that the new dependent variables $\tilde{\psi}_{n}(\vec{r}, t)$ satisfy the following system of nonlinear PDEs of Klein-Gordon type:

$$
\begin{aligned}
\tilde{\psi}_{n, t t} & -\Delta \tilde{\psi}_{n}-2 i \omega(\vec{r} \cdot \vec{\nabla}) \tilde{\psi}_{n, t}-i(2 \lambda+1) \omega \tilde{\psi}_{n, t} \\
& -\lambda(\lambda+1) \omega^{2} \tilde{\psi}_{n}-(2 \lambda+1) \omega^{2}(\vec{r} \cdot \vec{\nabla}) \tilde{\psi}_{n}-\omega^{2}(\vec{r} \cdot \vec{\nabla})^{2} \tilde{\psi}_{n} \\
& =2 \sum_{m=1, m \neq n}^{N}\left\{\frac{a-\left(\vec{\nabla} \tilde{\psi}_{n}\right) \cdot\left(\vec{\nabla} \tilde{\psi}_{m}\right)}{\tilde{\psi}_{n}-\tilde{\psi}_{m}}\right. \\
& \left.+\frac{\left[\tilde{\psi}_{n, t}-i \lambda \omega \tilde{\psi}_{n}-i \omega(\vec{r} \cdot \vec{\nabla}) \tilde{\psi}_{n}\right]\left[\tilde{\psi}_{m, t}-i \lambda \omega \tilde{\psi}_{m}-i \omega(\vec{r} \cdot \vec{\nabla}) \tilde{\psi}_{m}\right]}{\tilde{\psi}_{n}-\tilde{\psi}_{m}}\right\} .
\end{aligned}
$$

Note that this system is autonomous with respect to the time variable, but it features an explicit dependence on the space variable $\vec{r}$ (incidentally, in the last term in the left-hand side, $(\vec{r} \cdot \vec{\nabla})^{2} \equiv(\vec{r} \cdot \vec{\nabla})(\vec{r} \cdot \vec{\nabla})=S(\vec{r} \cdot \vec{\nabla})+\sum_{k, j=1}^{S} r_{k} r_{j} \nabla_{k} \nabla_{j}$, where of course $r_{j}$ denotes the $j$-th component of the $S$-vector $\vec{r}$ ); and it is clearly rotation invariant.

\section{Animations}

In this section we show a few solutions of the Schrödinger and Klein-Gordon type problems treated in the previous sections, displayed as animations over time.

Let us begin with a brief description of the methodology - implemented via a software written using Mathematica - employed to obtain the numerical results presented below. After assigning the space dimension $S$, the coefficients $\varphi_{n}(\vec{r}, t)$ (see (2.4) and (2.6)) and the monic polynomial $\Psi(\psi ; \vec{r}, t)$ of degree $N$ in $\psi$ (see $(2.1)$ ), we compute the $N$ zeros $\psi_{n}(\vec{r}, t)$ of this polynomial 

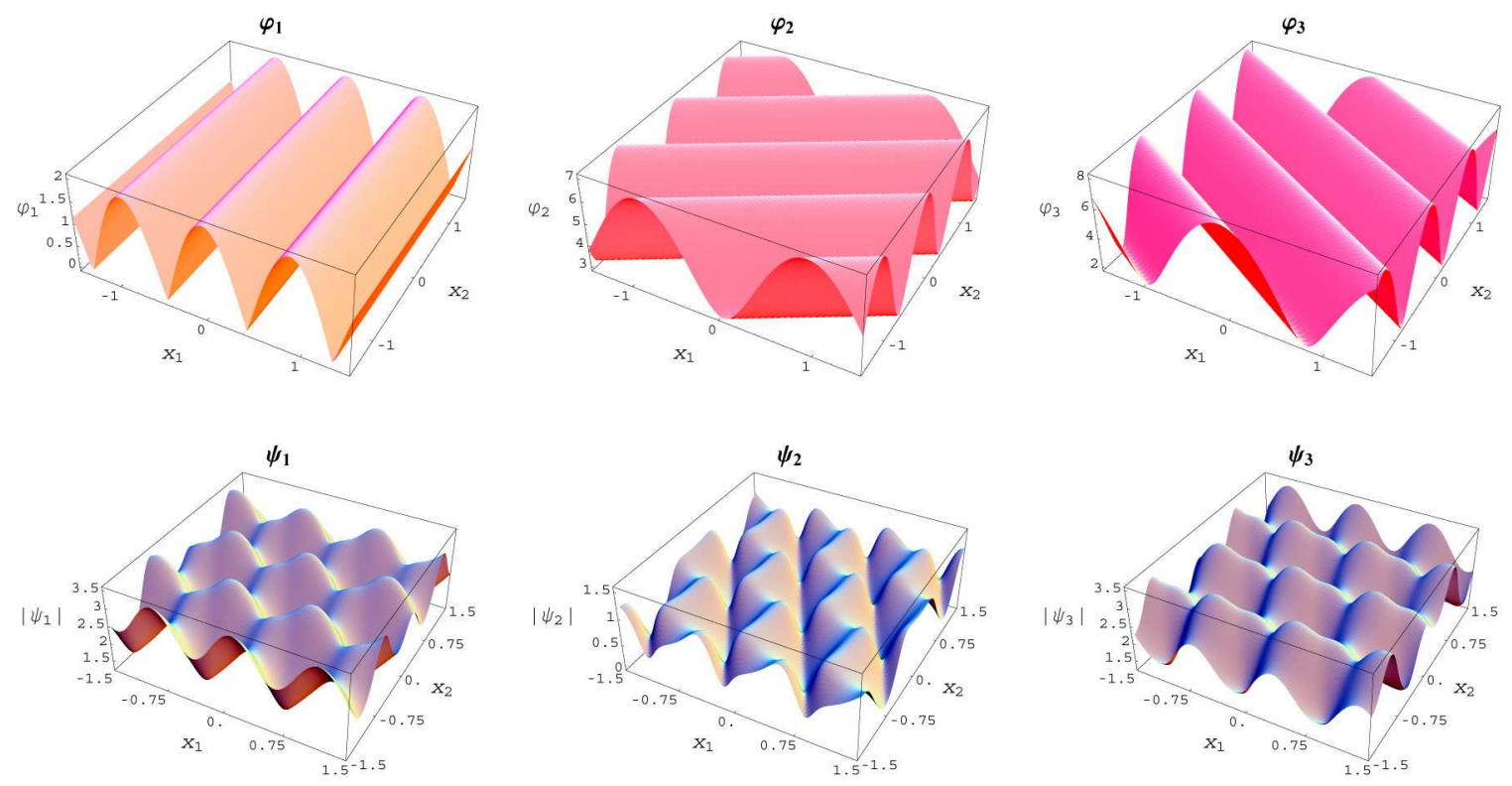

Figure 5.1.1. The $\varphi_{m}(\vec{r}, t)$ functions and the absolute values of the $\psi_{m}(\vec{r}, t)$ solutions for $0 \leq t \leq 1 / 8$.

as follows: firstly we create in the $(S+1)$-dimensional space of the independent variables $\vec{r}, t$ a lattice; next, we use a root-finding routine to calculate, at an appropriately chosen point of the lattice, the (generally complex) values of the $N$ zeros $\psi_{n}(\vec{r}, t)$ of the polynomial $\Psi(\psi ; \vec{r}, t)$, taking them in a generic order; and then we use an iterative root-finding procedure to calculate the $N$ zeros of the polynomial $\Psi(\psi ; \vec{r}, t)$ at any new point of the lattice, making use of the zeros previously calculated at the nearest points of the lattice so as to preserve the same initial ordering of the zeros.

In the following we consider only examples with $N=3$. The animations are organized as arrays of synchronized subanimations, showing above the time evolution of the three $\varphi_{m}(\vec{r}, t)$ functions, and below the corresponding time evolution of the three $\psi_{n}(\vec{r}, t)$ solutions. In each frame of the animation (namely, for a fixed value of the time variable), we display the values of the functions $\varphi_{m}(\vec{r}, t)$ and $\psi_{n}(\vec{r}, t)$ (or their absolute values $\left|\varphi_{m}(\vec{r}, t)\right|$ and $\left|\psi_{n}(\vec{r}, t)\right|$, when these functions are complex), with respect to the $x$ variable in two-dimensional plots if $S=1$, or with respect to the $x$ and $y$ variables in three-dimensional plots if $S=2$. In this paper we restrict attention only to cases with space dimension $S=1$ and $S=2$.

\subsection{Solutions of (1.1)}

In this subsection we present two numerical solutions of (1.1) displayed as animations, the first one with $S=2$ and the other two with $S=1$.

Example 5.1.1. The first animation corresponds to Example 3.3.1, with $S=2, a=b=0$, $W(r)=V(r)=0$ and the $\varphi_{m}(\vec{r}, t)$ functions as in (3.12), namely (but with 3 different 2-vector parameters $\left.\vec{v}_{m}\right)$

$$
\varphi_{m}(\vec{r}, t)=A_{m}+B_{m} \exp \left[-i \vec{v}_{m}\left(\vec{r}-\vec{v}_{m} t\right)\right]
$$

with

$$
A_{1}=1, \quad A_{2}=5, \quad A_{3}=-5,
$$



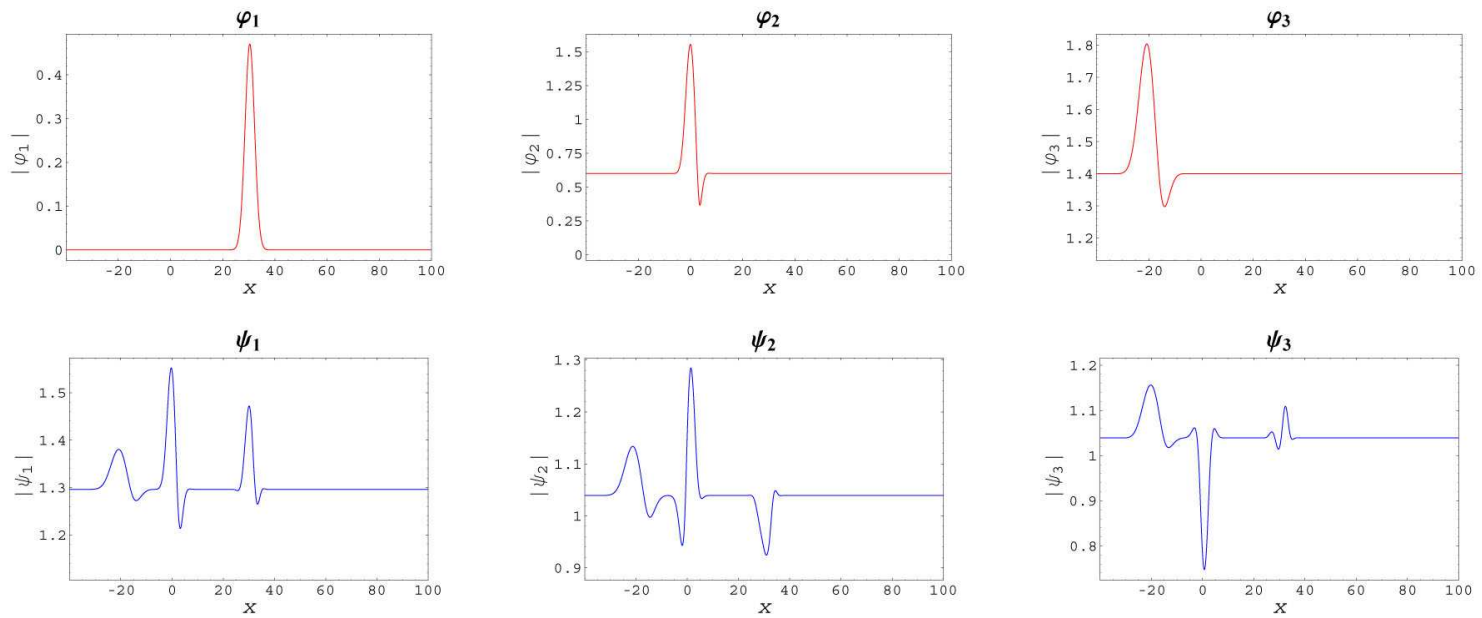

Figure 5.1.2. The absolute values of the $\varphi_{m}(x, t)$ functions and the absolute values of the $\psi_{m}(x, t)$ solutions for $0 \leq t \leq 20$.

$$
\begin{array}{lll}
B_{1}=1, & B_{2}=2, & B_{3}=3, \\
\vec{v}_{1}=(4 \sqrt{\pi}, 0), & \vec{v}_{2}=(-2 \sqrt{\pi}, 2 \sqrt{3 \pi}), & \vec{v}_{3}=(-2 \sqrt{\pi},-2 \sqrt{3 \pi}) .
\end{array}
$$

Here the periods in time of the three $\varphi_{m}(\vec{r}, t)$ functions are chosen to be the same, $T=1 / 8$, and the animation is performed on the time interval $0 \leq t \leq T$, then closed in loop.

Fig. 5.1.1 is the first frame. To see the whole animation, please click on the following (external) link: http://www.emis.de/journals/SIGMA/2006/Paper088/Animation5.1.1.gif.

Example 5.1.2. The second animation corresponds to the Example 3.3.1, with $S=1, a=b=$ $0, W(r)=V(r)=0$ and the $\varphi_{m}(x, t)$ functions as in (3.14), namely

$$
\varphi_{m}(x, t)=A_{m}+\frac{B_{m}}{\left(t+t_{m}+i \eta_{m}\right)^{1 / 2}} \exp \left[\frac{-i\left(x-x_{m}+i \eta_{m} v_{m}\right)^{2}}{4\left(t+t_{m}+i \eta_{m}\right)}\right],
$$

with

$$
\begin{array}{llllll}
A_{1}=0, & B_{1}=0.5, & x_{1}=30, & v_{1}=0.5, & \eta_{1}=1, & t_{1}=0.8, \\
A_{2}=-0.6, & B_{2}=-0.9, & x_{2}=0, & v_{2}=1, & \eta_{2}=2, & t_{2}=0.4, \\
A_{3}=1.4, & B_{3}=0.8, & x_{3}=-20, & v_{3}=0.5, & \eta_{3}=7, & t_{3}=1 .
\end{array}
$$

Here the animation is performed on the time interval $0 \leq t \leq 20$.

Fig. 5.1.2 is the first frame. To see the whole animation, please click on the following (external) link: http://www.emis.de/journals/SIGMA/2006/Paper088/Animation5.1.2.gif.

\subsection{Solutions of (1.2)}

In this subsection we present three numerical solutions of (1.2) displayed as animations, two with $S=2$ and the last with $S=1$.

Example 5.2.1. The first animation corresponds to the Example 4.3.1, with $S=2, a=b=0$, $M=0$ and the $\varphi_{m}(x, t)$ functions as in (4.4), with $K=4$ and a very particular choice of the functions $\varphi_{m}(x, y, t)$ :

$$
\varphi_{m}(x, y, t)=A_{m} \exp \left[-\frac{\left(x-x_{m}^{(1)}-t\right)^{2}}{a_{m}}\right]+B_{m} \exp \left[-\frac{\left(x-x_{m}^{(2)}+t\right)^{2}}{b_{m}}\right]
$$



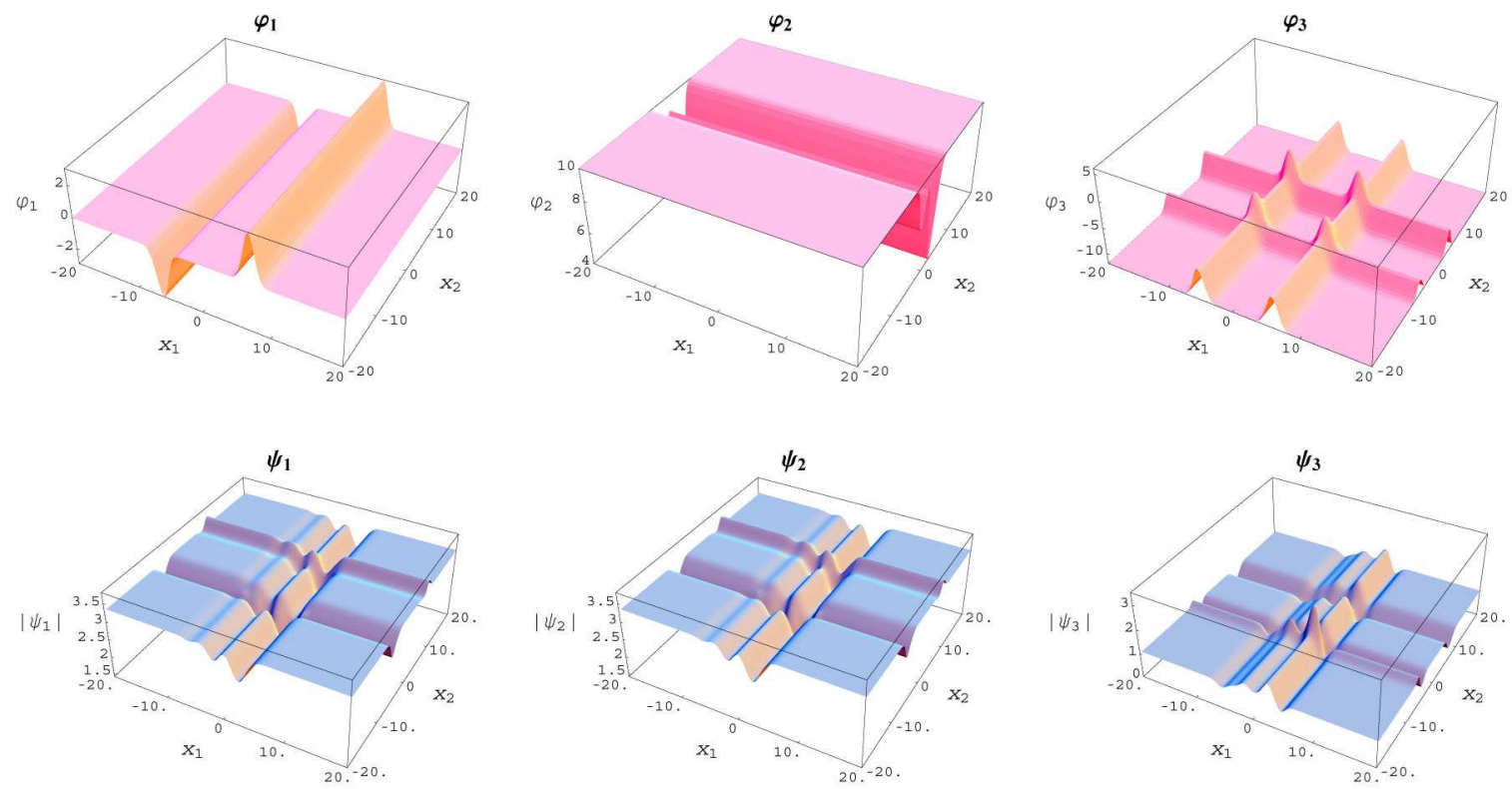

Figure 5.2.1. The $\varphi_{m}(\vec{r}, t)$ functions and the absolute values of the $\psi_{m}(\vec{r}, t)$ solutions for $0 \leq t \leq 20$.

$$
+C_{m} \exp \left[-\frac{\left(y-y_{m}^{(1)}-t\right)^{2}}{c_{m}}\right]+D_{m} \exp \left[-\frac{\left(y-y_{m}^{(2)}+t\right)^{2}}{d_{m}}\right]+E_{m}
$$

where

$$
\begin{array}{lllll}
A_{1}=-3, & B_{1}=3, & C_{1}=0, & D_{1}=0, & E_{1}=0, \\
A_{2}=0, & B_{2}=0, & C_{2}=-6, & D_{2}=-3, & E_{2}=10, \\
A_{3}=6, & B_{3}=6, & C_{3}=3, & D_{3}=4.5, & E_{3}=-12, \\
a_{1}=1.5, & b_{1}=1.3, & c_{1}=1.3, & d_{1}=1.6, & \\
a_{2}=1.8, & b_{2}=1.2, & c_{2}=2, & d_{2}=1.6, & \\
a_{3}=1.4, & b_{3}=1.5, & c_{3}=1.4, & d_{3}=1.2, & \\
x_{1}^{(1)}=-6, & x_{1}^{(2)}=7, & y_{1}^{(1)}=0, & y_{1}^{(2)}=0, & \\
x_{2}^{(1)}=0, & x_{2}^{(2)}=0, & y_{2}^{(1)}=2.5, & y_{2}^{(2)}=-1.5, & \\
x_{3}^{(1)}=-5, & x_{3}^{(2)}=6, & y_{3}^{(1)}=-5.5, & y_{3}^{(2)}=4.5 . &
\end{array}
$$

Here the animation is performed on the time interval $0 \leq t \leq 20$.

Fig. 5.2.1 is the first frame. To see the whole animation, please click on the following (external) link: http://www.emis.de/journals/SIGMA/2006/Paper088/Animation5.2.1.gif.

Example 5.2.2. The second animation corresponds to the Example 4.3.2, with $S=2, a=b=$ $0, M=0$ and the $\varphi_{m}(\vec{r}, t)$ functions as in (4.5):

$$
\varphi_{m}(x, y, t)=J_{0}\left(\sqrt{\left(x-x_{m}\right)^{2}+\left(y-y_{m}\right)^{2}}\right)\left[A_{m} \cos (t)+B_{m} \sin (t)\right]+C_{m},
$$

where

$$
A_{1}=1, \quad B_{1}=0.1, \quad C_{1}=0, \quad x_{1}=5, \quad y_{1}=0,
$$



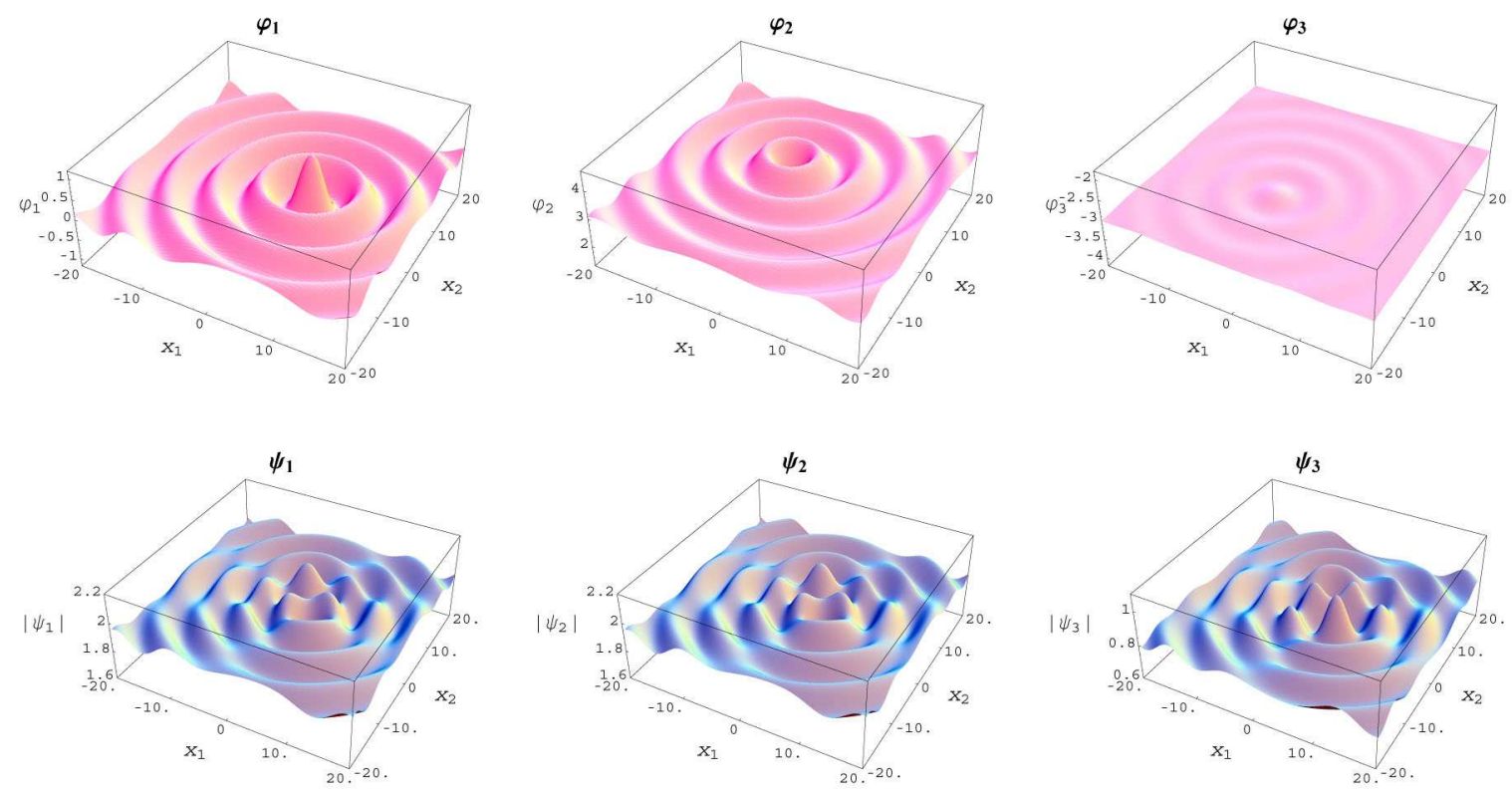

Figure 5.2.2. The $\varphi_{m}(\vec{r}, t)$ functions and the absolute values of the $\psi_{m}(\vec{r}, t)$ solutions for $0 \leq t \leq 2 \pi$.

$$
\begin{aligned}
& A_{2}=-1, \quad B_{2}=1, \quad C_{2}=3, \quad x_{2}=-5 / 2, \quad y_{2}=5 \sqrt{3} / 2, \\
& A_{3}=0.1, \quad B_{3}=1, \quad C_{3}=-3, \quad x_{3}=-5 / 2, \quad y_{3}=-5 \sqrt{3} / 2 \text {. }
\end{aligned}
$$

The $\varphi_{m}(\vec{r}, t)$ functions are periodic in time with period $T=2 \pi$. The animation is performed on the time interval $0 \leq t \leq T$, then closed in loop.

Fig. 5.2.2 is the first frame. To see the whole animation, please click on the following (external) link: http://www.emis.de/journals/SIGMA/2006/Paper088/Animation5.2.2.gif.

Example 5.2.3. The third animation corresponds again to the Example 4.3.1, with $S=1$, $a=b=0, M=0$ and the $\varphi_{m}(x, t)$ functions as in (4.4) with $K=2$, and with a very particular choice of the functions $\varphi_{m}(x, t)$ :

$$
\varphi_{m}(x, t)=A_{m} \cos \left(x-t+B_{m}\right)+C_{m} \cos \left(x+t+D_{m}\right)+E_{m},
$$

where

$$
\begin{aligned}
& A_{1}=1, \quad B_{1}=0, \quad C_{1}=3, \quad D_{1}=0.1, \quad E_{1}=0, \\
& A_{2}=2, \quad B_{2}=\pi, \quad C_{2}=-1, \quad D_{2}=\pi+0.1, \quad E_{2}=5, \\
& A_{3}=-1, \quad B_{3}=\pi / 2+0.1, \quad C_{3}=2, \quad D_{3}=\pi / 2, \quad E_{3}=-5 \text {. }
\end{aligned}
$$

The $\varphi_{m}(x, t)$ functions are periodic in time with period $T=2 \pi$ and periodic with respect to the $x$ variable with period $L=\pi$. The animation is performed on the time interval $0 \leq t \leq T$, then closed in loop.

Fig. 5.2.3 is the first frame. To see the whole animation, please click on the following (external) link: http://www.emis.de/journals/SIGMA/2006/Paper088/Animation5.2.3.gif.

\section{Outlook}

In future articles we plan to report additional investigations of these solvable (systems of) nonlinear evolution PDEs, and in particular to display visual animations of solutions of certain of these models in three-dimensional space. 


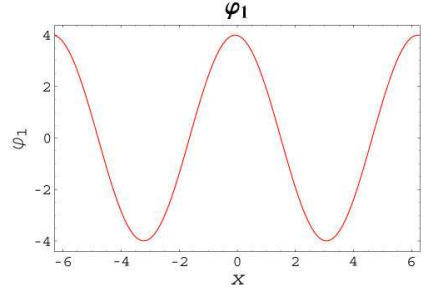

$\psi_{1}$

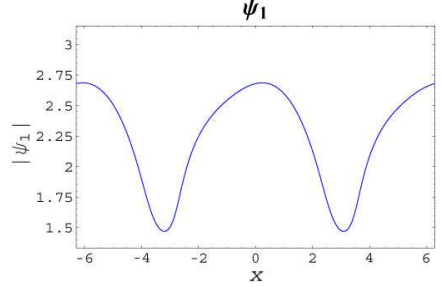

$\varphi_{2}$

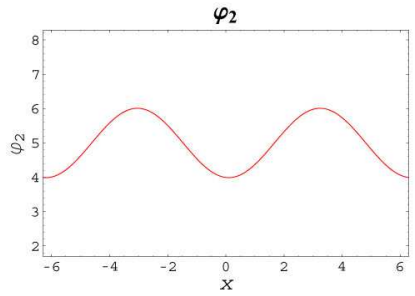

$\psi_{2}$

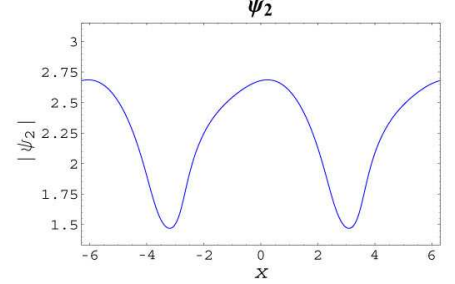

$\varphi_{3}$

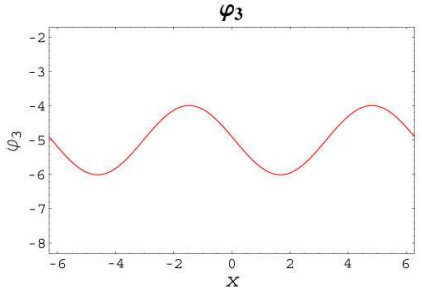

$\psi_{3}$

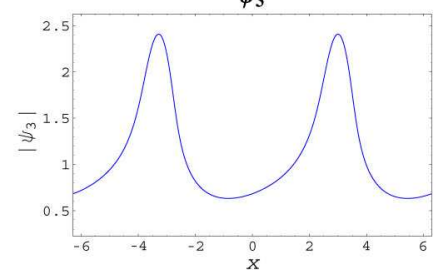

Figure 5.2.3. The $\varphi_{m}(x, t)$ functions and the absolute values of the $\psi_{m}(x, t)$ solutions for $0 \leq t \leq 2 \pi$.

\section{Acknowledgements}

The final correction of this paper was done while both authors were taking part in a Scientific Gathering on Integrable Systems and the Transition to Chaos at the Centro International de Ciencias in Cuernavaca.

[1] Calogero F., Motion of poles and zeros of special solutions of nonlinear and linear partial differential equations, and related "solvable" many body problems, Nuovo Cimento B, 1978, V.43, 177-241.

[2] Calogero F., A class of C-integrable PDEs in multidimensions, Inverse Problems, 1994, V.10, $1231-1234$.

[3] Calogero F., Classical many-body problems amenable to exact treatments, Lecture Notes in Physics Monograph, Vol. 66, Berlin Heidelberg, Springer-Verlag, 2001.

[4] Calogero F., Isochronous systems, Monograph, 200 pages, to appear.

[5] Erdélyi A. (Editor), Higher transcendental functions, Vol. II, New York, McGraw-Hill, 1953.

[6] Gómez-Ullate D., Sommacal M., Periods of the goldfish many-body problem, J. Nonlinear Math. Phys., 2005, V.12, suppl. 1, 351-362.

[7] Mariani M., Calogero F., Isochronous PDEs, Theor. Math. Phys., 2005, V.68, 958-968. 